\title{
Making changes: when is VVC appropriate?
}

\author{
Jerry Silverman, DVM
}

\begin{abstract}
"Why are we wasting energy trying to distinguish between a minor amendment and a major amendment?" asked Joanie Green, the new IACUC senior administrator at Great Eastern University. It seemed to her that if there was a request to add a procedure to a protocol it was to be considered an amendment, nothing more or less. "Are you saying that adding a small subcutaneous implant is as important as adding a cardiac valve replacement?" asked Bob Thorne, a clinical veterinarian. Green responded that it had nothing to do with the level of difficulty or risk to an animal, but whether or not there was a need for IACUC review.
\end{abstract}

"Look at it this way," she said, "if you add a subcutaneous implant as an experimental procedure, it's an amendment to the protocol and it gets reviewed by the IACUC. If you add a heart valve replacement to a study it's also an amendment that gets reviewed by the IACUC. The first is usually a minor procedure and the second is always a major procedure, but they're both amendments that require IACUC review."

After some additional discussion it became obvious that the real problem was that Thorne believed a "minor" procedure could be added to a protocol via the process of veterinary verification and consultation, as described in NIH notice NOT-OD-14-126 (ref. 1), if the IACUC had previously approved the methodology for the procedure. Green, on the other hand, was of the opinion that NOTOD-14-126 allowed for changes to be made by veterinary verification and consultation, but the addition of a new procedure, even a "minor" one, to a previously approved protocol was not a change and required IACUC review. What is your opinion?

1. National Institutes of Health. Guidance on Significant Changes to Animal Activities. Notice NOT-0D-14-126. (National Institutes of Health, Washington, DC, 26 August 2014).

\section{RESPONSE}

\section{Designated member review}

\section{Jeffrey J. Etue, BS, LATG}

This situation asks, "Can a new 'minor' procedure, such as a small subcutaneous implant, be added to the protocol via the process of VVC if the IACUC had previously approved the methodology for the procedure, or does the procedure require IACUC review?"

The Public Health Service Policy on Humane Care and Use of Laboratory Animals (PHS Policy; IV.B.7; ref. 1) requires that the IACUC "review and approve, require modifications in (to secure approval), or withhold approval of proposed significant changes regarding the use of animals in ongoing activities." The National Institutes of Health issued notice NOT-OD-14-126, Guidance on Significant Changes to Animal Activities ${ }^{2}$ to help IACUCs decrease the burden for full committee review (FCR) and designated member review (DMR) for some significant protocol changes by allowing veterinary verification and consultation (VVC).

According to NOT-OD-14-126 paragraph 2.a-c., the VVC can handle some significant protocol changes concerning "a. anesthesia, analgesia, sedation, or experimental substances; b. euthanasia to any method approved in the AVMA Guidelines for the Euthanasia of Animals; and c. duration, frequency, type, or number of procedures performed on an animal." 2 NOT-OD-14-126 does not define what constitutes a procedure. Thorne could argue that VVC could handle this amendment as the number of procedures performed on this animal will change to add a small subcutaneous implant. However, OLAW's online seminar "Implementing Guidance on Significant Changes: One Institution's Experience" states that procedures exclude surgeries. ${ }^{3}$

This small, subcutaneous implant amendment is addressed in paragraph 1.b., which covers changes "resulting in greater pain, distress, or degree of invasiveness" and possibly 1.e., which includes a "change in study objectives", of NOT-OD-14-126 (ref. 2); each of which require traditional IACUC review. Therefore, this amendment should go to the IACUC chairperson to decide on further action. Since the IACUC approved this type of procedure before, it may not warrant FCR but instead DMR could be appropriate. For Thorne, to go any further questioning review of this amendment appears erroneous and adding it by the process of VVC could lead to citations.

Research institutions should have a comprehensive policy in place compliant with NOT-OD-14-126 (ref. 2) to address amendments and enforce consultation with the IACUC chair for review. This would prevent the argument presented and any citations from the regulatory agencies.

1. Public Health Service. Policy on Humane Care and Use of Laboratory Animals (US Department of Health and Human Services, Washington, DC, 1986; amended 2002). 